EPJ Web of Conferences 64, 08003 (2014)

DOI: $10.1051 /$ epjconf/ 20146408003

(C) Owned by the authors, published by EDP Sciences, 2014

\title{
Observational Tests of Magnetospheric Accretion Models in Young Stars
}

\author{
Christopher M. Johns-Krull ${ }^{1,2,3, a}$ and P. Wilson Cauley ${ }^{1, b}$ \\ ${ }^{1}$ Rice University, Dept. of Physics \& Astronomy, Houston, TX 77005, USA \\ ${ }^{2}$ Visiting Astronomer, McDonald Observatory, Univ. of Texas at Austin, Austin, TX 78712, USA \\ ${ }^{3}$ Visiting Astronomer, NOAO, 950 N. Cherry Av., Tucson, AZ 85719, USA
}

\begin{abstract}
Magnetically controlled accretion of disk material onto the surface of Classical $\mathrm{T}$ Tauri stars is the dominant paradigm in our understanding of how these young stars interact with their surrounding disks. These stars provide a powerful test of magnetically controlled accretion models since all of the relevant parameters, including the magnetic field strength and geometry, are in principle measureable. Both the strength and the field geometry are key for understanding how these stars interact with their disks. This talk will focus on recent advances in magnetic field measurements on a large number of $\mathrm{T}$ Tauri stars, as well as very recent studies of the accretion rates onto a sample of young stars in NGC 2264 with known rotation periods. We discuss how these observations provide critical tests of magnetospheric accretion models which predict a rotational equilibrium is reached. We find good support for the model predictions once the complex geometry of the stellar magnetic field is taken into account. We will also explore how the observations of the accretion properties of the 2264 cluster stars can be used to test emerging ideas on how magnetic fields on young stars are generated and organized as a function of their internal structure (i.e. the presence of a radiative core). We do not find support for the hypothesis that large changes in the magentic field geometry occur when a radiative core appears in these young stars.
\end{abstract}

\section{Introduction}

T Tauri stars (TTSs), first classified by [1], are low mass, pre-main sequence (PMS) objects that are usually grouped into two classifications: 1. classical T Tauri stars (CTTSs), which show evidence of a circumstellar disk and mass accretion onto the central star in the form of excess emission in the X-ray, UV, optical, and infrared [2,3], and 2. weak-line T Tauri stars (WTTSs) which are also PMS stars but do not show evidence for significant mass accretion and do not have inner disks [4]. It is now well established that the excess emission observed in the spectra of CTTSs is due to the accretion of gas from a circumstellar disk [e.g. 5,6]. Magnetospheric accretion, in which gas from the disk accretes along stellar magnetic field lines, impacting the surface of the star at near free-fall velocities, explains many of the observed properties of CTTSs [7]. One important question is how CTTSs are able to shed angular momentum and rotate with velocities well below break-up [8,9,10,11]. The magnetospheric

\footnotetext{
ae-mail: cmj@rice.edu

be-mail: pwc1@ rice.edu
}

This is an Open Access article distributed under the terms of the Creative Commons Attribution License 2.0, which permits unrestricted use, distribution, and reproduction in any medium, provided the original work is properly cited. 
accretion model provides a potential answer to this question due to the role of the stellar magnetic field in transferring angular momentum from the star to the disk: if the magnetic field couples with a sufficiently ionized disk and acts as a braking torque on the star, it will rotate more slowly than if no braking mechanism were present $[12,13]$. This interaction essentially locks the star to the disk (i.e. disk-locking) and prevents the star from rotating at break-up velocity. This scenario is supported by the observed rotation rates of PMS stars with and without disks [9, 14-16]. However, contradictory results have been found in the ONC [17, 18] and NGC 2264 [11].

However, disk-locking models involve more than just the presence of a circumstellar disk. As discussed below, the equilibrium rotation rate in these models depend on the stellar mass, radius, and magnetic field and the disk accretion rate. As a result some of the ambiguity described above in the various rotation studies may be due to the large number of parameters that need to be considered. In an effort to account for the various parameters that enter the models, [19], used observations of 17 CTTSs in Taurus-Auriga to investigate correlations between the various parameters predicted by several different theories of magnetospheric accretion (see below) and found support for models that assume a non-dipolar surface field geometry and only weak support for models assuming purely dipolar fields. In this study, we extend the analysis of [19] to a larger sample of PMS stars in NGC 2264.

\section{Magnetospheric Accretion Predictions}

[20] examined the magnetspheric accretion theories of [12], [21], and [13] deriving equations for the stellar magnetic field $\left(B_{*}\right)$ in terms of $R_{*}, P_{r o t}, \dot{M}$, and $M_{*}$. One of the main assumptions underlying these accretion theories is that the central star is magnetically locked to its disk so that the star rotates at the Keplerian velocity of the disk truncation radius. By looking at the correlations predicted between stellar and accretion parameters by these magnetospheric accretion theories we can test the validity of these models as applied to PMS stars. The relationships predicted by [12] and [13] depend in the same way on the stellar and accretion parameters:

$$
R_{*}^{3} B_{d i p} \propto M_{*}^{5 / 6} \dot{M}^{1 / 2} P_{r o t}^{7 / 6} .
$$

Predictions based on [21] deviate only slightly from eq. (1), and produce nearly identical results in plots [19]. Therefore, we only display plots generated using eq. (1) when considering the [12], [21], and [13] models with a dipolar field.

Standard magnetospheric accretion models all assume that the stellar magnetic field is purely dipolar. Observations of magnetically sensitive absorption lines in TTSs, however, have shown that most TTSs have complex surface field geometries [22-26]. [19] show that if the dipolar field requirement is relaxed, but that the amount of magnetic flux participating in the accretion flow is conserved and mapped back to the stellar surface from the truncation point, then the model of [13] can be modified to predict the following relationship:

$$
R_{*}^{2} f_{a c c} B_{a c c} \propto M_{*}^{1 / 2} \dot{M}^{1 / 2} P_{r o t}^{1 / 2}
$$

where $f_{a c c}$ is the filling factor of the accretion columns on the stellar surface, i.e. the percentage of the surface covered by accretion flows. $B_{a c c}$ is now the strength of the field participating in the accretion flow at the surface of the star. The magnetic flux participating in the accretion flow is equal to one-third of the total magnetic flux trapped at the truncation point [27]. Equation (2) is a specific prediction of [19]'s modified analysis of the [27] theory of magnetospheric accretion.

Equations (1) and (2) are directly testable if measurements of the stellar and accretion parameters can be obtained. Estimates for $f_{a c c}$ can be obtained by modeling the accretion flow as a hot slab of 


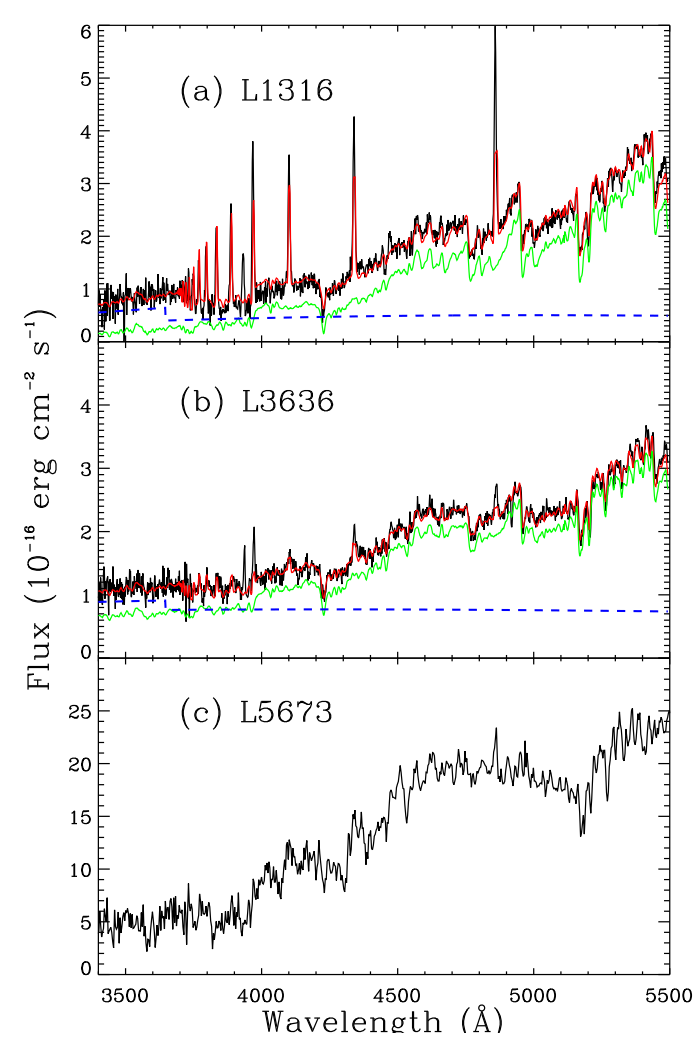

Figure 1. Example spectra and their associated models. Panel (a) shows L1316 (designations come from [10], a moderately accreting star (several stars are more strongly accreting that this); panel (b) shows L3636, a weakly accreting star; panel (c) displays L5673, a typical star in which we find no evidence for accretion. The black lines are the observed spectrum; the final model spectrum is overplotted in red; the underlying template star spectrum is overplotted in green and the slab spectrum (with the Balmer lines excluded for clarity) is overplotted with a blue dashed line. Models are not produced for the non-accreting stars.

emitting hydrogen $[6,28]$. Due to the lack of magnetic field measurements for stars in NGC 2264, we are not able to substitute measured values of the surface magnetic field into eqs. (1) and (2). On the other hand, measurements of mean surface magnetic fields on CTTSs have shown the field strengths to be relatively constant from star to star [20, 23, 29-31]. Therefore, we proceed by first making the simple assumption that the dipolar component of the magnetic field (eq. 1) and the magnetic field participating in the accretion flow (eq. 3) are constant from star to star. Because we are concerned only with how well the two sides of eqs. (1) and (2) are correlated, assuming a constant field strength allows us to ignore $B_{d i p}$ and $B_{a c c}$ in the analysis.

\section{Observations and Data Reduction}

Our sample consists of 36 pre-main sequence stars of spectral type K and M in the open cluster NGC 2264, and 14 main sequence dwarfs ranging from spectral type K1 to M4 which serve as spectral templates. The sample is a subset of objects from the rotation study of [10], and the stellar identification numbers are those used in that study. In the end, only eight of the observed templates are actually used in our models. The NGC 2264 targets were chosen based on the availability of a previously determined spectral type and rotation period, and their confirmed PMS evolutionary nature based on approximate $\mathrm{H} \alpha$ equivalent widths. All of the stars in our sample are brighter than $\mathrm{I}<16.0$.

We obtained low resolution ( $R \sim 600)$ optical (3000 $\AA-6000 \AA$ ) spectrophotometry of each of our target stars at either McDonald Observatory using the $2.7 \mathrm{~m}$ Harlan J. Smith telescope, or at Kitt Peak National Observatory using the $4 \mathrm{~m}$ Mayall telescope. The wavelength resolution of the 

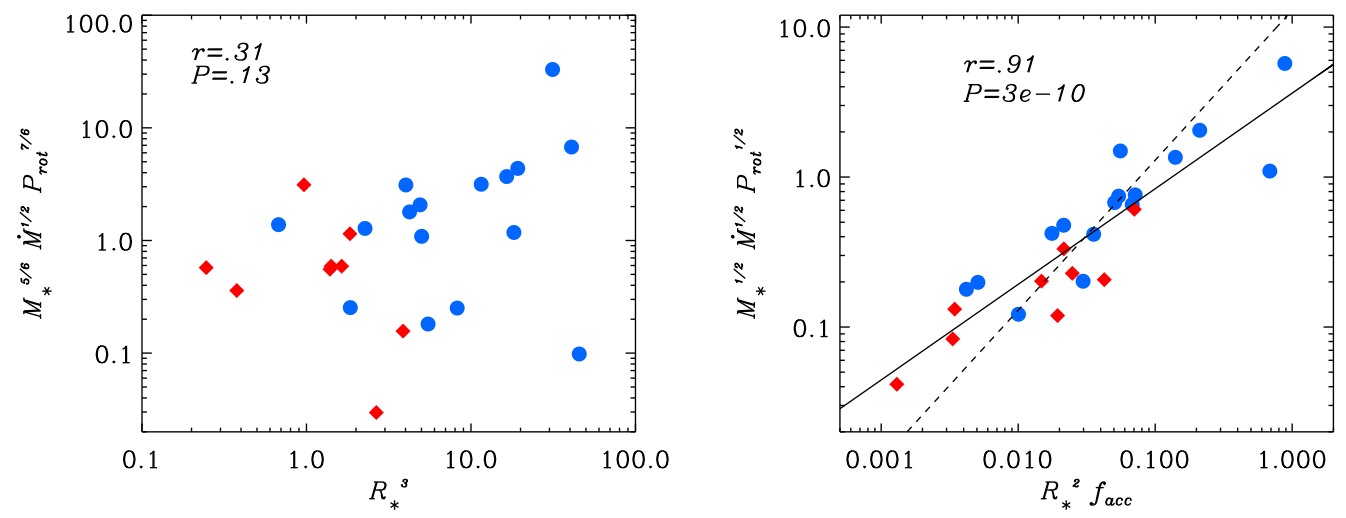

Figure 2. The left panel plots the right hand side of eq. (1) versus the left hand side of eq. (1), and the right panel plots the right hand side of eq. (2) versus the left hand side of eq. (2). In both cases it is assumed the magnetic field strength does not vary from one star to the next. The red diamonds show the weakly accreting stars in the sample similar to the middle panel of Fig. 1 and the blue circles show strongly accreting stars similar to the top panel of Fig. 1. The value of the correlation coefficient, $r$, and the associated false alarm probability, $P$, is given in each panel.

McDonald observations is $\sim 7.2 \AA$; for the Kitt Peak observations $\Delta \lambda \sim 3.2 \AA$. Spectrophotometric flux standards taken from [32] and [33] were observed each night at various airmasses in order to generate atmospheric extinction curves and flux conversion factors. Typical exposure times for the McDonald sample were $\sim 1000$ seconds with a signal to noise ratio of $\sim 90-100$ at $4400 \AA$, approximately the center of the modeled wavelength region and free of any significant emission lines. Exposure times for the Kitt Peak sample were typically $\sim 1200-1800$ seconds with a signal to noise ratio of $\sim 40-50$ at $4400 \AA$ A. Signal-to-noise ratios for fainter Kitt Peak stars (I>15) are closer to $\sim 20$. Typically the S/N ratio of the data decreases towards bluer wavelengths as the detector becomes less sensitive to these photons. All of the data were reduced using standard procedures via custom IDL routines written for this purpose. The reductions include wavelength calibration, correction for atmospheric extinction, and flux calibration. Uncertainties for each spectrum were also calculated including the effects of CCD read noise and Poisson noise from the source and the sky.

\section{Analysis}

We follow the same procedure as in [28] to model the observed spectra of the NGC 2264 PMS stars. Briefly, each PMS star spectrum is modeled as the sum of the spectrum from hydrogen slab in LTE added to the spectrum of a template star of the appropriate spectral type. This summed spectrum is then reddened and scaled to match the observed spectrum. The free parameters of the hydrogen slab include its temperature, density, thickness, and the filling factor $\left(f_{a c c}\right)$ it occupies on the stellar surface. The additional free parameters of the model are the reddening to the star and the overall scale factor which takes into account the distance to the source. A non-linear least squares method is employed to find the best fit model. Such a hydrogen slab model has been found to be a good representation of the bulk of the emission from the accretion zones [6, 28]. Once a final fit is determined for a given PMS star, the parameters of the slab can be used to compute its spectrum over all wavelengths which 
can then be integrated and combined with the distance to NGC 2264 of 760 pc [34] to determine the accretion luminosity which can then be used to calculate the mass accretion rate. The result of this modeling is then a measure of both $\dot{M}$ and $f_{a c c}$ which are needed to test the models described above. The stellar mass and radius come from placing the objects in the HR diagram and using the evolutionary tracks of [35]. Example fits to three of our observed NGC 2264 stars is shown in Figure 1. See [34] for fits to all the stars and further details of the analysis.

With the accretion stellar and accretion parameters from the spectral fitting, we can then use the rotation periods measured by [10] to test the relationships given in eqs. (1) and (2). These comparisons are shown in Figure 2, which clearly shows that the correlation predicted by eq. (1) is not present in the data, while the correlation predicted by eq. (2) does appear to be realized at some level by the observations of these young stars in NGC 2264. A total of 24 stars have sufficient measurements to be included in these plots. A linear regression fit to the observed points (solid line in Figure 2) does have a somewhat different (shallower) slope compared to the predicted correlation (the dashed line in the right hand panel of Figure 2) from eq. (2). [19] also found a shallower slope in this relationship for their study in Taurus-Auriga.

\section{Conclusion}

The comparisons shown in Figure 2 offer very little support for disk-locking theory in young stars for the case where the magnetic field is assumed to be a pure dipole (the left hand panel). The model of [27] offers a prescription for abandoning the dipole assumption, instead just keeping track of the stellar magnetic flux that is participating in the accretion flow. The observed behavior of the sample of 24 PMS stars studied here in NGC 2264 show a strong correlation between their stellar, accretion, and rotation parameters when compared to the predictions of this non-dipole model. This can be interpreted as strong support for the model of [27]. However, [36] find that the stellar magnetic flux participating in the accretion flows in their numerical simulations which assume a dipole stellar field is very similar to that found by [27] when the star is near spin equilibrium. It may then be that the key point is that since the dipole component of the magnetic field falls off the least rapidly with distance, the interaction of the field with the disk at the truncation radius is well described by all the disk-locking models described in $\$ 2$ as long as the stellar magnetic field considered is only the dipole component. The lack of correlation in the left hand panel of Figure 2 might then suggest that the dipole component varies strongly from one star to the next.

In addition to testing magnetospheric disk-locking models in young stars, our data can also be used to examine an emerging paradigm in the evolution of magentic field properties of newly formed stars. It had been suggested, based on magnetic maps of only a handful of stars, that while PMS stars are fully convective they have strong dipole components to their magnetic fields, and soon after a radiative core develops the dipole weakens substantially [e.g. 37]. Under this scenario, once a star develops a radiative core and its dipole component weakens, the accretion disk will be able to push closer in to the stellar surface and the disk-locked equilibrium rotation rate will increase. The exact rotation rate will still depend on the actual accretion rate and stellar properties as before, just with the dipole component of the field weakening. Thus, we can use the equations in [20] to calculate the expected value of $B_{d i p}$ for each of our stars and see if this value correlates with the appearance of a radiative core. Doing this we find no correlation at all, and therefore, no support for this new paradigm in our data [for details, see 34]. This may indicate that the paradigm is not correct, or the sample of stars we have observed is not in a disk-locked mode. 
EPJ Web of Conferences

\section{References}

[1] A. H. Joy, ApJ 102, 168 (1945)

[2] C. Bertout, ARAA 27, 351 (1989)

[3] C. Argiroffi, A. Maggio, \& G. Peres, A\&A 465, L5 (2007)

[4] F. M. Walter, F. M., PASP 99, 31 (1987)

[5] C. Bertout, G. Basri, \& J. Bouvier, ApJ 330, 350 (1988)

[6] P. Hartigan, et al., ApJ 382, 617 (1991)

[7] J. Bouvier, et al. "Magnetospheric Accretion in Classical T Tauri Stars", Protostars and Planets $V$, eds. Reipurth, B., Jewitt, D., \& Keil, K. (The University of Arizona Press, Tucson, AZ, USA 2007) $479-494$

[8] S. N. Vogel\& L. V. Kuhi, ApJ 245, 960 (1981)

[9] W. Herbst, et al., A\&A 396, 513 (2002)

[10] M. H. Lamm, et al., A\&A 417, 557 (2004)

[11] R. B. Makidon, et al., AJ 127, 2228 (2004)

[12] A. Königl, ApJ 370, L39 (1991)

[13] Shu, F., et al., ApJ 429, 781 (1994)

[14] S. Edwards, et al., AJ 106, 372 (1993)

[15] K. E. Kearns \& W. Herbst, AJ 116, 261 (1998)

[16] P. I. Choi\& W. Herbst, AJ 111, 283 (1996)

[17] K. G. Stassun, R. D. Mathieu, T. Mazeh, \& F. J. Vrba, AJ 117, 2941 (1999)

[18] L. M. Rebull, AJ 121, 1676 (2001)

[19] C. M. Johns-Krull \& A. D. Gafford, ApJ 573, 685 (2002)

[20] C. M. Johns-Krull, J. A. Valenti, \& C. Koresko, ApJ 516, 900 (1999)

[21] A. C. Cameron \& C. G. Campbell, A\&A 274, 309 (1993)

[22] C. M. Johns-Krull, et. al., ApJ 510, L41 (1999)

[23] J. A. Valenti \& C. M. Johns-Krull, Ap\&SS 292, 619 (2004)

[24] A. G. Daou, C. M. Johns-Krull \& J. A. Valenti, AJ 131, 520 (2006)

[25] J.-F. Donati, et al., MNRAS 386, 1234 (2008)

[26] J.-F. Donati, et al., MNRAS 412, 2454 (2011)

[27] E. Ostriker \& F. H. Shu, ApJ 447, 813 (1995)

[28] J. A. Valenti, G. Basri, \& C. M. Johns, AJ 106, 2024 (1993)

[29] E. W. Guenther, H. Lehmann, J. P. Emerson, \& J. Staude, A\&A 341, 768 (1999)

[30] C. M. Johns-Krull, ApJ 664, 975 (2007)

[31] H. Yang, \& C. M. Johns-Krull ApJ, 729, 83 (2011)

[32] M. Hamuy, et al., PASP 104, 533 (1992)

[33] J. B. Oke, AJ 99, 1621 (1990)

[34] P. W. Cauley, et. al., ApJ 756, 68 (2012)

[35] L. Siess, E. Dufour, \& M. Forestini, A\&A 358, 593 (2000)

[36] C. Zanni \& J. Ferreira, A\&A 550, 99 (2013)

[37] J.-F. Donati, et al. MNRAS 417, 472 (2011) 